\title{
Description et point de vue : un travail de lecture/ écriture au collège
} Marie-Christine Vinson

\section{Citer ce document / Cite this document :}

Vinson Marie-Christine. Description et point de vue : un travail de lecture/ écriture au collège. In: Pratiques : linguistique, littérature, didactique, $n^{\circ} 55,1987$. Les textes descriptifs. pp. 89-99;

doi : https://doi.org/10.3406/prati.1987.1453

https://www.persee.fr/doc/prati_0338-2389_1987_num_55_1_1453

Fichier pdf généré le 13/07/2018 


\title{
DESCRIPTIONS ET POINT DE VUE : UN TRAVAIL DE LECTURE/ÉCRITURE AU COLLĖGE
}

\author{
Marie-Christine VINSON
}

\begin{abstract}
Faire travailler le point de vue à travers l'apprentissage du texte descriptif "réaliste" permet d'aborder deux notions essentielles à la lecture/écriture des textes dans une relation qui n'a rien d'artificiel. En effet les enjeux de la description sont assumés par le narrateur/descripteur qui la conduit. Mais choisir de travailler description et point de vue, c'est aussi vouloir utiliser dans la classe les outils que proposent la grammaire textuelle et la typologie des textes sur des énoncés que la narratologie moderne domine. Les travaux de Ph. Hamon sur la description sont là pour le prouver.
\end{abstract}

\section{I - PRÉSENTATION DE L'ACTIVITÉ D'ÉCRITURE DANS LAQUELLE S'INSCRIT LE TRAVAIL}

Une classe de troisième de 23 élèves est engagée dans l'écriture de microromans comme le reste des classes du collège, d'ailleurs, qui conduit un projet d'écriture longue (1). La $3^{\text {e }} 4$ a choisi le genre roman autobiographique, ce choix ayant peut-être été guidé en partie par la lecture de "L'Enfant " de Jules Vallès qui se déroulait à ce moment là. Mais à l'instar de tous les élèves du collège, il a été décidé que l'on écrirait sur le quartier. En tant qu'usagers (élèves/habitants) d'un haut-lieu médiatique, les Minguettes, ils veulent écrire leur propre ville qui a souvent été racontée par d'autres, les journalistes. Le roman autobiographique nécessite la présence d'un je narrateur qui assure la narration ; les situations et les personnages pourront être réels/fictifs (il ne s'agit pas de "se mettre à nu" dans son texte) mais le lieu, lui, doit être identifiable. Alors pour faire exister les Minguettes dans le texte que les élèves sont en train de rédiger, il faut l'écrire et rapidement se pose le problème de la description. Puisqu'il s'agit de parler d'un lieu réel, d'en donner une vision personnelle mais aussi de faire vrai en rendant ce lieu reconnaissable, le modèle de description choisi est celui de la description "réaliste". Ce modèle est également justifié par le genre d'écrit dans lequel il doit s'insérer, le roman autobiographique. Immédiatement se superpose une deuxième difficulté : le lieu étant vu par le je du roman autobiographique, comment gérer le point de vue dans le texte descriptif ? Ces problèmes se posent à peu près au même moment du travail d'écriture pour tous les élèves. Très vite les groupes de scripteurs (la possibilité d'écrire seul ou à deux avait été donnée dès le début) sont amenés à parler du lieu dans lequel ils situent les événements de leur récit. C'est donc de cette activité limitée mais faisant partie d'un projet plus global, la réalisation de micro-romans, que nous voudrions rendre compte.

(1) Voir Pratiques n० 52, "Pratiques de lecture" 


\section{Mise en route du travail}

Tout d'abord est proposé à la classe un petit exercice oral qui permet, de façon simple et concrète, de dégager les traits spécifiques du texte descriptif. Deux élèves vont l'un après l'autre à la fenêtre et décrivent ce qu'ils voient. Chacun peut vérifier en regardant à son tour par la fenêtre que les élèves descripteurs ont choisi certains éléments parmi tous ceux qui s'offraient à leurs yeux et qu'ils les ont présentés dans un certain ordre, organisant ainsi leur commentaire. De la sorte se trouve posé le conflit existant entre la simultanéité de la vision de l'objet (toutes les parties sont vues en même temps) et la successivité de la parole ou de l'écriture obéissant au principe de la linéarité. Se trouve également posée l'activité sélective du descripteur :Abdérazak n'a pas retenu exactement les mêmes éléments que Safina. Écrire une description demande donc de savoir régler le choix et l'organisation des éléments saisis par le je descripteur.

Mais le recensement des demandes et des besoins ne s'arrête pas là. La description va devoir trouver sa place dans le récit que les élèves sont en train de rédiger. Intervient ici ce que Ricardou appelle la " belligérance" du texte (2). Comment, en effet, arrêter le cours du récit? Comment justifier par rapport à la narration la présence de la description? Comment la camoufler pour qu'elle n'apparaisse pas comme un arrêt brutal et intempestif du récit?

Une fois posés les problèmes à résoudre, il s'agit de mettre en place des stratégies d'écriture qui explicitent au maximum les protocoles à suivre pour que chaque scripteur puisse avancer dans sa propre production.

\section{II - UNE PREMIÈRE APPROCHE DU TEXTE DESCRIPTIF ET DU POINT DE VUE}

\section{1 - Est distribué à chaque élève de la classe le texte suivant :}

"L'officier ouvrit vivement la fenêtre, comme si l'oxygène eût manqué à ses poumons, et il vint respirer, sur un large balcon, cet air pur que distillait une belle nuit de juillet.

Sous ses yeux, baignée par les rayons lunaires, s'arrondissait une enceinte fortifiée, dans laquelle s'élevaient deux cathédrales, trois palais et un arsenal. Autour de cette enceinte se dessinaient trois villes distinctes, Kitaï-Gorod, Beloï-Gorod, Zemlianoï-Gorod, immenses quartiers européens, tartares ou chinois, que dominaient les tours, les clochers, les minarets, les coupoles de trois cents églises, aux dômes verts, surmontès de croix d'argent. Une petite rivière, au cours sinueux, rèverbérait çà et là les rayons de la lune. Tout cet ensemble formait une curieuse mosaïque de maisons diversement colorées, qui s'enchâssait dans un vaste cadre de dix lieues.

Cette rivière, c'était la Moskowa, cette ville, c'était Moscou, cette enceinte fortifiée, c'était le Kremlin, et l'officier des chasseurs de la garde qui, les bras croisés, le front songeur, écoutait vaguement le bruit jeté par le Palais-Neuf sur la vieille cité moscovite, c'était le czar" (3).

Cet extrait court, structuré en trois paragraphes est rapidement identifié comme une description: il est donc soumis à l'analyse et à l'interrogation commune.

Le premier paragraphe ne parle pas encore du paysage vu mais plutôt de celui qui voit. Sa fonction introductive est repérée ainsi que les traits spécifiques qui la composent. Hormis le personnage qui voit (l'officier), les élèves relèvent un lieu d'observation (fenêtre, large balcon), un prétexte (comme si l'oxygène eût

(2) J. Ricardou, Le texte en conflit in Nouveaux problèmes du roman, Seuil, 1978, p. 24 et suivantes. (3) Jules Verne. Miche/ Strogoff, Livre de poche, 1978, p. 15 et 16. 
manqué à ses poumons, il vint respirer), des conditions favorables qui permettent au regard de s'exercer (air pur, belle nuit de juillet). Ainsi tout le premier paragraphe fonctionne comme une sorte de mise en scène; la description (travail du scripteur) est montrée comme l'affaire du personnage, ce qui permet regarde).

- de justifier la pause narrative

- d'assurer le point de vue (un militaire en position dominante dans l'espace

Le deuxième paragraphe est alors étudié. Au préalable, il est demandé aux élèves de dessiner, de façon schématique bien sûr, le paysage. Par ce biais, il s'agit de faire comprendre la mise en espace et la structuration de la vision.

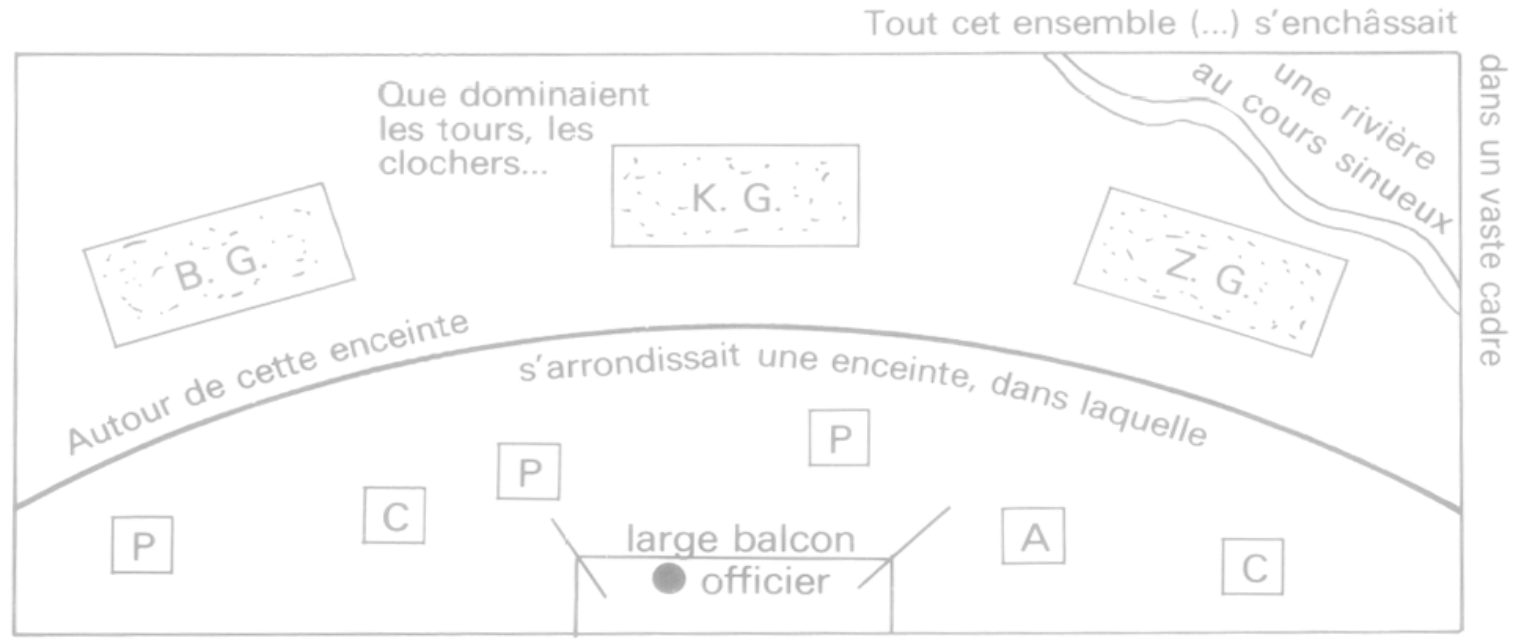

Le schéma oblige à une lecture attentive et favorise le repérage des mots et expressions qui permettent une localisation des éléments. Le schéma permet aussi de relativiser la nécessité de localiser. Très vite les élèves se rendent compte de l'absence d'exhaustivité en ce domaine. Si les deux cathédrales, les trois palais et l'arsenal se trouvent dans l'enceinte, aucune indication n'autorise à situer l'arsenal sur la droite, sur la gauche ou en face du descripteur. Cette remarque n'est pas isolée : il en est de même pour les trois villes, la rivière au cours sinueux. En fait la description de type réaliste n'est pas un calque de réel, n'est pas une photographie mais doit produire un effet de réel, doit susciter l'adhésion du lecteur pour un décor vraisemblable.

Après les problèmes relatifs à la mise en espace, est abordé le choix des éléments retenus pour décrire le paysage. Le regard du descripteur ne s'attarde et ne détache que les éléments qui représentent un enjeu de pouvoir. II est alors demandé aux élèves de dresser la liste des "choses vues" et de caractériser les pouvoirs qu'elles symbolisent (l'enceinte fortifiée, l'arsenal : le pouvoir militaire ; le palais : le pouvoir politique ; les cathédrales, clochers, minarets... : le pouvoir religieux). Les précisions numériques (deux cathédrales etc...) connotent la puissance et la rigueur du plan de campagne. Le texte confirme lui-même cette lecture : si les trois villes "distinctes" sont mentionnées, les maisons qui les composent ne font l'objet d'aucun commentaire particulier. Quant aux notations de couleur et de lumière, elles sont peu nombreuses et peu diversifiées. Là encore les couleurs ne sont précisées que lorsqu'elles signalent un élément symbole de pouvoir (dômes verts, croix d'argent).

Les verbes utilisés doivent aussi faire l'objet d'une attention particulière : ils donnent des indications de forme ou de situation (ex. : s'arrondissait...). Les élé- 
ments du paysage en devenant sujets de ces verbes, "s'animent". Cela permet de dynamiser la description et de minimiser le statisme qu' elle suscite par rapport au reste du récit qui assure la relation des péripéties.

La lecture en classe peut encore être approfondie. On repère l'alternance des lignes horizontales et verticales qui structurent le passage (ex. : $\mathrm{H}=$ enceinte fortifiée ; $V=s^{\prime}$ élevaient deux cathédrales, trois palais, un arsenal ; $H=$ trois villes distinctes...). Ainsi se donne-t-on les moyens de montrer l'enjeu de l'organisation de la description qui, ici, met en valeur les points stratégiques du paysage vus par l'officier de sorte que l'objet décrit apparaît comme un plan militaire.

Enfin le troisième paragraphe est abordé : sa fonction est d'accroître la production de l'intérêt romanesque. La devinette est levée. Comme dans le jeu des métiers, les deux premiers paragraphes faisaient s'interroger : qui est-ce ? ou estce? L'identité est alors dévoilée : la rivière, c'est la Moskowa... l'officier, c'est le czar. Cette fois, une nouvelle description est proposée mais précise et synthétique, elle s'oppose à la description analytique du paragraphe précédent et en permet une lecture rétroactive. Toute cette stratégie renforce la position dominante de celui qui voit :

- il est dominant dans l'espace, le paysage est à ses pieds.

- il est dominant politiquement, militairement, c'est le czar

- il est dominant sémiotiquement (au niveau du système qui produit le sens) car il sait (dès le début) que la petite rivière s'appelle la Moskowa...

\section{2 - Quelques exercices d'écriture}

Ce premier travail terminé, il peut être "consolidé " par une série d'exercices d'écriture assez courts qui favorisent les processus d'appropriation des notions expliquées.

1. Dans le texte qui a servi à l'analyse, changer le descripteur: ce n'est plus le czar qui regarde mais une dame de la cour qui vient se rafraîchir après la danse, c'est un domestique qui s'accorde un temps de repos, c'est un peintre...

Une autre variante de ce type de manipulation peut être proposée : selon le principe d'Exercices de Sty/e, décrire un objet, toujours le même, en faisant varier le point de vue.

2. Prendre un texte et demander aux élèves d'insérer une description à l'endroit qui convient le mieux. Il s'agit de chercher, en s'appuyant sur une lecture précise, le personnage qui peut jouer le rôle du descripteur, de repérer les éléments existants susceptibles d'être utilisés dans l'introduction du texte descriptif c'est-àdire d'assurer le passage narration/description. II faut aussi cerner le point de vue du personnage qui voit et déterminer ce qu'il regarde. Voici à titre d'exemple le début du $2^{\circ}$ chapitre de la Sixième de Susie Morgenstern (4).

"Margot partit enfin, inquiète de paraître si peu à la mode avec un cartable, terrifiée à l'idée d'avoir des profs sévères, tracassée par la crainte qu'ils se soient trompés et qu'il n'y ait pas de place pour elle en sixième. Sa mère l'accompagna.

Les enfants et les parents ètaient debout dans la cour du collège. Margot cherchait des yeux des visages familiers. Elle vit deux copines de son ancienne école et se dirigea à leur rencontre. Soudain il y eut un mouvement vers le préau. Une voix autoritaire commanda aux parents de rester à l'extérieur du préau et aux enfants de se regrouper silencieusement à l'intérieur : mais les parents restaient collés. Un monsieur rond et moustachu lisait les noms de la sixieme 1 : "Si vous ne vous taisez pas, il vous faudra revenir demain ou après-demain, moi ça m’est égal ".

(4) Susie Morgenstern, La Sixieme. Ecole des Iousirs, 1984. p. 20 et 21. 
rayès des listes!"

Margot se concentrait tres fort. Elle avait peur de louper son nom, peur qu'on ne la nomme pas, peur presque d'oublier son nom qu'elle se répéta pour le garder en tête".

La description interviendra après la phrase "Margot cherchait des yeux des visages familiers". Margot sera celle qui regarde. Le fait qu'elle cherche des yeux des visages familiers justifiera le texte descriptif en donnant au descripteur un prétexte pour observer. C'est la rentrée des classes, la fillette découvre le collège et surtout la cour, son regard sera celui du novice inquiet qui appréhende un lieu nouveau. "Elle vit deux copines de son ancienne école et se dirigea à leur rencontre" clora la description puisque les retrouvailles font disparaître la raison même de l'observation.

\section{III - DEUXIĖME APPROCHE : AFFINER ET APPROFONDIR LA NOTION DE POINT DE VUE A TRAVERS LE TEXTE DESCRIPTIF}

Ces premières réflexions, si elles permettent de lancer l'écriture des élèves ne règlent pas tous les problèmes. Certains dysfonctionnements font apparaître que la notion de point de vue n'est pas assez maîtrisée. Le texte de Nathalie en offre un exemple:

"Tout en roulant, $M$. et Mme Lecomte et leur fille observèrent les lieux avec curiosité. A travers la vitre de la voiture, on distinguait, sur leur droite, quatre grandes tours qui leur paraissaient gigantesques vues d'en bas. Elles se ressemblaient beaucoup et avaient les mêmes formes rectangulaires, la seule différence était que l'une était blanche alors que les trois autres étaient marron. Tout autour se dessinaient d'immenses terrains verts que le soleil faisait étinceler tandis qu'à leur gauche, on apercevait distinctement des pâtés de maisons alignées de façon régulière. Plus loin s'élevaient des tours de quinze étages qui dominaient les Minguettes. A l'horizon, on apercevait les cheminees de Feyzin qui polluaient l'astmosphère..

ville"

Ce cadre etait assez impressionnant pour des personnes étrangères à la

La stratégie du regard choisi est celle du point de vue des nouveaux (les Lecomte). Mais immédiatement, il y a glissement vers un point de vue anonyme "on" (générique) c'est-à-dire le narrateur (dans ce cas précis, il ne s'agit pas du je autobiographique). De plus l'ambiguité de "leur" (on distinguait sur leur droite) est intéressante à souligner. "Leur" renvoie aux Lecomte mais corrélé avec "on", "leur" fait que les Lecomte deviennent objet et non plus sujet assurant la description. Le point de vue se trouve en quelque sorte occulté et on ne sait qui prend en charge les images. La non-maîtrise formelle (Lecomte/ils/on) suppose que tout le monde a le même regard et surtout entraîne la suppression des clivages: "Je décris le monde comme il est". La confusion entre la construction médiate du texte et la reproduction immédiate du réel provoque un effet d'uniformisation et d'appauvrissement.

\section{La notion de point de vue}

Le point de vue fait alors l'objet d'un nouveau travail avec les èlèves. La typologie en trois points que présente G. Genette dans Figures III, donne à l'enseignant des outils de réflexion. Utilisant le terme de focalisation (plus abstrait et moins visuel), il propose:

- la focalisation zéro, le narrateur en dit plus que n'en sait aucun des personnages, c'est le narrateur omniscient (narrateur > personnage). 
- la focalisation interne, le narrateur ne dit que ce que sait tel personnage (narrateur $=$ personnage).

- la focalisation externe, le narrateur en dit moins que n'en sait le personnage (narrateur spersonnage).

Mais au niveau de l'usage en classe, on peut se contenter d'un dispositif allégé. Nous reprenons la proposition de J.P. Goldenstein qui, dans Pour lire le roman (5) donne une typologie à deux points: vision illimitée, vision limitée.

\subsection{La vision illimitée}

Dans le cas de la vision illimitèe, le descripteur est omniscient, il voit tout. II peut décrire des lieux différents, éloignés dans l'espace et dans le temps. La description peut être assurée soit par un "je", soit par une troisième personne sans identité précise.

II s'agit par exemple de l'incipit d'Eugènie Grandet. La grande description d'ouverture dresse le décor : sont présentés Saumur et les personnages, Grandet et sa maisonnée. Le descripteur omniscient (troisième personne sans identité précise) renvoie même, par anticipation, à la suite du texte pour plus de précisions descriptives.

"La description des autres portions du logis se trouvera liée aux événements de cette histoire ;..." (6).

\subsection{La vision limitée}

Le descripteur présente le point de vue d'un personnage lié à l'action. Cette vision se trouve donc limitée à ce que le personnage voit, entend, connaît. Un extrait de Lucien Leuwen peut être soumis à la lecture de la classe avec la consigne de repérer les marques qui signalent la vision subjective.

"Lucien leva les yeux et vit une grande maison, moins mesquine que celles devant lesquelles le régiment avait passé jusque-là ; au milieu d'un grand mur blanc, il y avait une persienne peinte en vert perroquet. "Quel choix de couleurs voyantes ont ces marauds de provinciaux!" Lucien se complaisait dans cette idée peu polie lorsqu'il vit la persienne vert perroquet s'entrouvrir un peu ; c'était une jeune femme blonde qui avait des cheveux magnifiques et l'air dédaigneux : elle venait voir défiler le régiment. Toutes les idées tristes de Lucien s'envolèrent à l'aspect de cette jolie figure; son âme en fut ranimée. Les murs écorchés et sales des maisons de Nancy, la boue noire, l'esprit envieux et jaloux de ses camarades, les duels nécessaires, le méchant pavé sur lequel glissait la rosse qu'on lui avait donnée, peut-être exprès, tout disparut." (7).

Les verbes "leva les yeux", "vit" désignent Lucien comme le descripteur. Toute une série d'adjectifs qualificatifs, de noms précise le point de vue du personnage (mesquine, vert perroquet, marauds, magnifique, dédaigneux...). L'exclamative au discours direct met en avant le jugement personnel.

Après cette mise au point qui peut sembler un peu formelle, il paraît intéressant de travailler avec la classe les combinaisons et les effets produits par le point de vue dans un texte descriptif.

(5) Pour lire le roman. De Boek Duculot, 1986, p. 33 et suivantes

(6) H. de Balzac, Eugénie Grandet, Garnier-Flammarion, 1964, p. 43

(7) Stendhal, Lucien Leuwen, Folio, 1973, p. 97 


\section{Combinaisons et effet du point de vue dans le texte descriptif}

Le point de vue peut changer à l'intérieur d'une œuvre, d'une séquence, d'un même segment narratif. Ses jeux et ses variations permettent des effets qui influent sur la lecture. Montrer comment est utilisé le point de vue dans certaines descriptions, $n^{\prime}$ améliore pas seulement les compétences en lecture mais aussi les compètences en écriture en ouvrant le champ des possibles descriptifs.

\subsection{Le point de vue contradictoire}

L'exemple cité par A. Petitjean dans Pratiques d'écriture (8) nous semble particulièrement éclairant à ce sujet. Dans Germinal, à une vingtaine de pages d'intervalle, s'opposent le peuple vu par les bourgeois et les bourgeois vus par le peuple. Les deux descriptions se répondent et mettent en valeur les représentations que chacun a de l'autre, sur fond de peur ou d'envie. Là encore, faire lire les élèves avec un crayon, en leur demandant de souligner tous les indices qui servent à construire le point de vue, peut être un travail profitable.

\subsection{Le point de vue formel}

II arrive que le narrateur se camoufle derrière un personnage/descripteur, prétexte qui sera mis ostensiblement en avant. On peut s'interroger en classe sur l'intérêt d'une telle stratégie et sur les répercussions dans le fonctionnement du texte. Nous avons choisi le début de La Terre de Zola qui, par son rôle de "scène d'exposition", pose des problèmes intéressants (9).

"Jean, ce matin-là, un semoir de toile bleue noué sur le ventre, en tenait la poche ouverte de la main gauche, et de la droite, tous les trois pas, il y prenait une poignée de blé, que d'un geste, à la volée, il jetait. Ses gros souliers trouaient et emportaient la terre grasse dans le balancement cadencé de son corps ; tandis que, à chaque jet, au milieu de la semence blonde toujours volante, on voyait luire les deux galons rouges d'une veste d'ordonnance, qu'il achevait d'user. Seul, en avant, il marchait, l'air grandi, et, derrière, pour enfouir le grain, une herse roulait lentement, attelée de deux chevaux, qu'un charretier poussait à longs coups de fouet réguliers, claquant au-dessus de leurs oreilles.

La parcelle de terre, d'une cinquantaine d'ares à peine, au lieu dit des Cornailles, était si peu importante, que M. Hourdequin, le maître de la Borderie, n'avait pas voulu y envoyer le semoir mécanique, occupé ailleurs. Jean, qui remontait la pièce du midi au nord, avait justement devant lui, à deux kilomètres, les bâtiments de la ferme. Arrivé au bout du sillon, il leva les yeux, regarda sans voir, en soufflant une minute.

$C^{\prime}$ étaient des murs bas, une tache brune de vieilles ardoises, perdue au seuil de la Beauce, dont la plaine, vers Chartres, s'étendait. Sous le ciel vaste, un ciel couvert de la fin d'octobre, dix lieues de cultures étalaient en cette saison les terres nues, jaunes et fortes, des grands carrés de labour, qui alternaient avec les nappes vertes de luzernes et des tréfles; et cela sans un coteau, sans un arbre, à perte de vue, se confondant, s'abaissant, derrière la ligne d'horizon, nette et ronde comme sur une mer (....). Des villages faisaient des îlots de pierre, un clocher au loin émergeait d'un pli de terrain, sans qu'on vît l'église, dans les molles ondulations de cette terre du blé".

Dans les deux premiers paragraphes, la description est assurée par le narrateur montrant Jean au travail. Dès la fin du deuxième paragraphe, il est facile de repérer la mise en place du dispositif qui va placer Jean en position de descripteur. Selon les critères définis avec les élèves lors de l'étude du premier texte (I'extrait tiré de Michel Strogoff), nous retrouvons ici:

- un lieu d'observation (le paysage ouvert)

(8) A. Petitjean, Pratiques d'écrizure, Cedic, 1982, p. 88 et 89

(9) E. Zola, La Terre, Le livre de poche, 1979, p. 7, 8 et 9 
minute).

- un prétexte pour marquer une pause et justifier la vision (en soufflant une

- des verbes de perception qui désignent celui qui regarde (levant les yeux, regarda sans voir).

Pourtant dans le troisième paragraphe, la description est-elle vraiment assurée par Jean? La question se pose. II n'y a pas de verbes de perception (examiner, observer) qui permettent un ancrage du point de vue, il n'y a pas non plus d'indice révélateur de la subjectivité du regard (ex.: sembler). Par contre, à la fin du paragraphe, apparaît un " on " (sans qu' on vît l'église) qui n'est pas sans rappeler le "on" des deux premiers paragraphes (on voyait luire...). En poursuivant la lecture (nous n'avons pas reproduit ici la suite du texte, il suffit de se reporter aux trois premières pages du livre de poche), se trouvent confirmées les fluctuations sur le descripteur. Jean est mis en avant (il avait devant lui), puis réapparition du on (on ne devinait les prairies, on se trouvait là dans l'ancien Dumois) enfin retour du regard de Jean (il jeta un coup d'œil en bas). Les mêmes remarques peuvent être formulées à propos du cinquième paragraphe. Quant au sixième paragraphe, il place de nouveau Jean en position d'observateur (il aperçut, lui fait de nouveau lever la tête). La fin de la description est marquée au niveau de la narration par la présence du dialogue, au niveau de la fiction par l'intervention de Françoise tirée par la Coliche, perturbation qui permet au récit de commencer.

Le personnage prétexte (Jean) mis en avant parce qu'il va devenir le héros de l'histoire et intervenir dès le début du roman, sert à vraisemblabiliser le texte descriptif, à le "faire passer". En fait le narrateur investit la totalité de la description. Il faut dresser un tableau complet et précis du décor (fonction de la scène d'exposition) et donner au lecteur une série de compétences administratives (I'ancien Dunois devenu aujourd'hui l'arrondissement de Châteaudun...), agricoles (l'assolement triennal...), et surtout géographiques (le Perche opposé à la Beauce, les vallons à la plaine, les richesses de la Beauce fertiles aux duretés de la Beauce pouilleuse). L'influence du milieu sur l'homme que postule Zola n'est pas étrangère à cette stratégie.

\subsection{Le point de vue personnel}

Alors que dans le début de La Terre, le personnage/descripteur disparaît au profit du narrateur et du paysage décrit, dans La Curée, la description intervient comme support et/ou prétexte pour faire connaitre le personnage (10).

"Quand le garçon fut sorti, Renée prit son binocle et fit curieusement le tour du petit salon. C'était une pièce carrée, blanc et or, meublée avec des coquetteries de boudoir. Outre la table et les chaises, il y avait un meuble bas, une sorte de console où l'on desservait, et un large divan, un véritable lit qui se trouvait placé entre la cheminée et la fenêtre. Une pendule et deux flambeaux Louis XVI garnissaient la cheminée de marbre blanc. Mais la curiosité du cabinet était une glace, une belle glace trapue que les diamants de ces dames avaient criblée de noms, de dates, de vers estropiés, de pensées prodigieuses et d'aveux étonnants. Renée crut apercevoir une saleté et n'eut pas le courage de satisfaire sa curiosité. Elle regarda le divan, éprouva un nouvel embarras, se mit, afin d'avoir une contenance, à regarder le plafond et le lustre de cuivre doré, à cinq becs. Mais la gêne qu'elle ressentait était délicieuse. Pendant qu'elle levait le front, comme pour étudier la corniche, grave et le binocle à la main, elle jouissait profondément de ce mobilier équivoque qu' elle sentait autour d'elle ; de cette glace claire et cynique, dont la puretè, à peine ridée par ces pattes de mouche ordurières, avait servi à rajuster tant de faux chignons; de ce divan qui la choquait par sa largeur; de la table, du tapis lui-même, où elle retrouvait l'odeur de l'escalier, une vague odeur de poussière pénétrante et comme religieuse".

(10) E. Zola, La Curée, Le fivre de poche, 1979, p. 202 et 203. 
Renée découvre un lieu interdit et le plaisir de la transgression. Plusieurs fois, dans le cours du texte, des verbes qui signalent le point de vue rappelant que le cabinet est regardé par Renée: "Renée prit son binocle et fit curieusement le tour du petit salon", "Renée crut apercevoir", " et n'eut pas le courage", "éprouva un nouvel embarras", "la gêne qu"elle ressentait était délicieuse", " elle jouissait profondément de ce mobilier". Tous les éléments vus sont saturés de subjectivité : le mobilier est équivoque, la glace est claire et cynique, le divan est choquant par sa largeur, la table, le tapis dégagent une odeur pénétrante. La description exprime l'état d'esprit du personnage et ce qu'il ressent.

Le point de vue personnel peut déboucher sur un jeu de démultiplication. Si les élèves poursuivent leur lecture de La Curée, les pages suivantes montrent le même personnage, Renée, regardant à trois moments différents le même paysage par la fenêtre. Les deux premières descriptions ont lieu avant l'adultère, la dernière, juste après. La substitution d'un type discursif à un autre, le descriptif prenant la place du narratif, permet de raconter l'adultère sans le montrer directement. L'analyse faite en classe, montrera comment, dans une sorte de jeu métonymique, description et point de vue traduisent :

- la naissance du désir, le vocabulaire du feu et des flammes domine dans le premier texte (11) où Maxime et Renée "s' abandonnent à la griserie des bruits et des clartés" en regardant les filles,

- la volupté, dans le deuxième texte (12) !e regard de Renée transforme l'extérieur en intérieur (les lumières s'éteignent, les prostituées raccolent les derniers clients, les bruits s'apaisent), Renée voit la rue comme une invitation à la tendresse,

- la honte, dans le troisième texte (13), Renée fait de la rue maintenant plongée dans l'ombre, une alcôve solitaire et renvoie sur la ville pour la partager, la honte qu'elle ressent.

Dans chacune de ces descriptions, Renée remarque une prostituée vêtue de bleu, attendant et résignée. Elle est comme le double du personnage qui regarde (Renée porte un ruban bleu), sorte de préfiguration de l'avenir où Renée sera rejetée par Maxime.

Le travail de lecture et d'analyse fait sur les différents extraits que nous venons de présenter a permis aux élèves de se constituer un dossier de textes (qu'ils pouvaient augmenter par des recherches personnelles), véritable outil dans les travaux d'écriture/réécriture parallèles.

\section{IV - ANALYSE DE TRAVAUX D'ÉLĖVES}

Écritures et lectures de textes ont avancé, les difficultés rencontrées en écrivant sollicitant de nouvelles lectures et analyses. Les trois textes qui vont suivre ont été écrits par un groupe de filles, Magalie et Sylvie, et représentent trois moments différents de l'acquisition du texte descriptif à travers un travail d'amélioration.

\section{État I}

"Je regardais le soleil se coucher sur les Minguettes qui etaient ma ville natale, je pensais à la journée qui m'attendait le lendemain".

La description est embryonnaire, on peut même dire inexistante. II n'y a pas d'introduction justificative: " je" regarde mais le lecteur ne sait pas pourquoi. En

(11) ibid., p. 203, 204, 205, 206

(12) ibid., p. 210 et 211

(13) ibid., p. 215,216 et 217 
fait, l'objet décrit se réduit à un seul mot "Minguettes". C'est à partir de ce constat et des problèmes posés par l'écriture d'un tel type de texte qu'a débuté l'étude de la description.

\section{État II}

"Je regardais le soleil se coucher derrière ces blocs de bèton qui m'étaient familiers. L'immeuble, en face de chez moi, était vert et d'un blanc qui virait sur le gris. Une rangée de jeunes peupliers séparait le parking qui commençait à se remplir de voitures de touies sortes de couleurs, de la pelouse verdoyante où je pouvais apercevoir des chiens surveillés par l'œil attentif de leur maittre. Certaines allées étaient éclairées. Des volets se fermaient. J'entendais des pleurs d'enfants, des rires, des chuchotements, des roulements de billes sur le sol... Je jetais un dernier coup d'cil avant de fermer mes volets en pensant à la journée qui m'attendait le lendemain."

Tout de suite, on peut noter une amélioration quantitative qui est aussi qualitative. L'introduction est encore à reprendre : il n'y a pas de raison, de prétexte qui justifie la pause narrative et l'observation du descripteur. Par contre l'objet décrit a pris forme. Plusieurs éléments ont été choisis pour évoquer le paysage : l'immeuble, les jeunes peupliers, le parking, les voitures, la pelouse... Des verbes de perception jalonnent le texte (apercevoir, entendre, jeter un coup d'œil). Des notations de couleur (verdoyance, blanc, gris...) ainsi que sonores (bruits de volets, cris...) montrent la maîtrise de certains procédés par le groupe de scripteurs. Si l'arrêt de la description est signalé (fermeture des volets), il n'est pas assez justifié.

Remarquons également que le "je" qui regarde commence à manifester son point de vue. L'impression d'un quartier paisible où dominent les activités de la vie quotidienne crée un ensemble rassurant (familier, chiens surveillés, des rires, des chuchotements, roulement de billes). Mais il faut accentuer l'effet de bonheur tranquille que veulent produire les élèves. De plus il n'y a aucun travail sur la mise en espace: on ne relève peu ou pas d'adverbes ou de locutions adverbiales.

\section{État III}

“En attendant ma mère, je regardais à travers les vitres de ma chambre le soleil se coucher derrière ces blocs de bètons qui m'étaient très familiers. J'étais née aux Minguettes. L'immeuble, en face de chez moi, était vert et d'un blanc qui virait sur le gris. Une rangée de jeunes peupliers séparait la pelouse verdoyante du parking qui, à cette heure avancèe, était rempli de voitures. Le chien du voisin du dessous dèambulait sous l'œil attentif de son maître. Plus loin, certaines allées étaient déjà éclairées. Au dessous, des volets se fermaient, peut-être ceux de Mme Bonnaccueil. l'entendais monter des pleurs d'enfants, des rires, des chuchotements, des roulements de billes sur le sol...

Je jetais un dernier coup d'œil dans la rue Claude Debussy pour voir si ma mère arrivait. Puis je me décidais à fermer les volets en pensant à la journée qui m'attendait le lendemain".

Cette fois les éléments fournis par les mises au point théoriques sont presque tous assimilés. La description est précisément justifiée: I'attente de la mère et le désœuvrement conduisent le descripteur à la fenêtre. La mise en espace de l'objet décrit est effective (en face, plus loin, au-dessus...). Et surtout le point de vue assuré par le " je" s'est précisé. Une réflexion sur le lien entre paysage rassurant et paysage connu a permis aux élèves de travailler à partir du mot "familier". L'expression "ville natale" qui se trouvait dans l'état I a été reprise après modification pat "j'étais née aux Minguettes" jugé plus explicite. C'est toujours pour donner une impression de connu que certains éléments se sont trouvés modifiés. "Des chiens" sont devenus "le chien du voisin du dessous", "Mme Bonnaccueil a fermé les volets". L'onomastique elle-même a été sollicitée pour traduire la tranquillité du 
lieu. D'ailleurs ce travail sur l'onomastique a dépassé le cadre de la description et s'est étendu au reste du texte. Le groupe a modifié les noms des personnages : Félicité Bonheur est devenu le nom du "je" narrateur, Joi, Aimée et Baraka, ceux des principaux protagonistes. En examinant ce troisième état du texte, on peut s'apercevoir que la fermeture $d \leqslant$ la description s'est elle aussi améliorée. II y a reprise des éléments du début (je jetais un dernier coup d'œil, pour voir si sa mère arrivait) et justification de l'interruption (fermer les volets en pensant à la journée qui $m$ ' attendait le lendemain), ce qui va permettre de reprendre la narration et de poursuivre le récit.

Le travail présenté moritre comment lecture et écriture peuvent être sollicitées pour construire une démarche d'apprentissage. Le va-et-vient textes/productions permet a'aller chercher daris les livres des réponses aux problèmes qui se posent dans l'écriture.

Le travail d'un point particulier (description et point de vu!e) inséré dans un projet global (la rédaction d'un récit autobiographique) est justifié. II ne s'agit plus de décrire pour sacrifier à un rituel scolaire mais d'apprendre à décrire parce que c'est une nécessité pour faire avancer le projet. II ne s'agit plus de décrire pour décrire mais de choisir le type de description le mieux adapté, de rendre compte de son organisation et de son articulation avec le point de vue.

L'explicitation précise des critères d'analyse facilite le repérage des dysfonctionnements. Ainsi l'évaluation des écrits et les remarques qui en découlent, sont cernées, les réécritures progressives rendues possibles.

\section{INDICATIONS BIBLIOGRAPHIQUES}

Ph. Hamon, "Qu'est-ce qu'une description?" in Poétique n" 22, 1972.

$\mathrm{Ph}$. Hamon, "Un discours contraint" in Littérature et réalité, Seuil, 1982.

R. Barthes, "L'effet de réel" in Littérature et réalité, Seuil 1982.

J.P. Goldenstein, “Un récit d'initiation: Michel Strogoff" in Pratiques $m^{\prime \prime 22 / 23, p . ~}$ 49 et suivantes, repris dans Pour une lecture-écriture, de Boeck Duculot, 1985.

J.P. Goldenstein, "Comment ? La représentation de I'espace romanesque " in Pour lire le roman, de Boeck Duculot, 1986, p. 91 et suivantes.

A. Petitjean, "La description" in Pratiques d'écriture, Cedic, 1982, p. 81 et suivantes. 